\title{
Dynamics based on analysis of public data for spreading of disease
}

\author{
Leonardo S. Lima ${ }^{1}$ \\ ${ }^{1}$ Federal Education Center for Technological of Minas Gerais, 30510-000, Belo Horizonte, MG, Brazil. \\ *Islima@cefetmg.br/Islima7@yahoo.com.br
}

\begin{abstract}
The stochastic model for epidemic spreading of the novel coronavirus disease based on the data set supported by the public health agencies in countries as Brazil, EUA and India is investigated. We performed the numerical analysis using the stochastic differential equation for estimating of the novel cases diary as well as analytical calculations solving the correspondent partial equation for the distribution of novel cases $P$. Our results display that the model based in the ltô diffusion fits well to the results diary due to uncertain in the official data and to the number of tests realized in the populations of each country.
\end{abstract}

\section{Introduction}

The coronavirus disease (COVID-19) emerged in China in the end of 2019 year and since then it has spread reaching all countries in the globe. The quick spreading of the disease made the study of its temporal evolution of great importance for forecasting, control and prevention. ${ }^{1-5}$ On the other hand, the modelling of natural phenomena up to end of nineteenth century can be viewed as the study of deterministic solutions of differential equations where at that time thought that if all initial data could only be collected, one would be able to predict the future with certainly. We now know this is not so since that the concept of chaos has arisen, in which even quite simple differential equation systems have the rather property of giving rise to unpredictable behavior. A historical example is the Brownian motion or the motion viewed of pollen grains in suspension in the fluid, discovered by the British botanic Robert Brown who observed firstly the motion in 1827. It was not quickly solved, and a satisfactory explanation did not come until 1905 by Einstein. The same explanation was independently developed by Smoluchowski. The Einstein's results were derived later by Langevin who writes in the form of the following differential equation $d x / d t=a(x, t)+b(x, t) \xi(t)$, where $a(x, t)$ and $b(x, t)$ are certain known functions and $\xi(t)$ is the rapidly fluctuating random term. In general, an idealised mathematical formulation of the concept of a highly irregular function is that for $t \neq t^{\prime}$, $\xi(t)$ and $\xi\left(t^{\prime}\right)$ are statistically independent. Furthermore, we also require $\langle\xi(t)\rangle=0$, since any nonzero mean can be absorbed into the definition of $a(x, t)$, and thus require that $\left\langle\xi(t)\left\langle\xi\left(t^{\prime}\right)\right\rangle=\delta\left(t-t^{\prime}\right)\right.$, which satisfies the requirement of no correlation at different times and has the rather result that $\xi(t)$ has infinite variance. The Langevin equation is so the first type of a stochastic differential equation. An derivation in a foot mathematically more rigorous of the Langevin equation was performed later by the mathematicians Itô and Stratonovich. ${ }^{6}$

It is known that the logistic model of population growing can be used as model for some infectious diseases as the smallpox that are spread largely by individuals who can transmit the disease but who exhibit no overt symptoms. ${ }^{7}$ The logistic model may be modified so that unbounded growth does not occur. The simplest way to do this is to introduce a factor that will have the effect of making the variation rate of population growing $\wp, d \wp / d t$ negative when $z$ is large. Where the model is given in form $d \wp / d t=-\alpha \wp(1-v \wp)(1-\eta \wp)$, with $\alpha$ and $\eta$ parameters such that $\alpha>0$ and $0<\eta<v$. Furthermore, we can allow some randomness in these parameters with aim to obtain a more realistic model of real situation. ${ }^{8-12}$ In general, if the size of the population at time $t$ is $\wp(t)$ and $\varpi(t)$ being the relative rate of growth at time $t$, we can have $\varpi(t)$ subjects to some random due to environmental effects, so that $\varpi(t)=\wp(t)+\xi(t)$, where the first term $\wp(t)$ is deterministic while the second term $\xi(t)$ reflects to the environmental randomness effect. Particularly, if $\xi(t)$ presents the properties $\langle\xi(t)\rangle=0$ and $\left\langle\xi(t) \xi\left(t^{\prime}\right)\right\rangle=\Gamma \delta\left(t-t^{\prime}\right)$ with $\Gamma$ constant being the amplitude of the white noise.

In this paper, one investigates the stochastic diffusion model as possible model for spreading of the COVID-19. The use of this type of analysis has already been employed in the study of the behavior of time series for price dynamics in Refs. ${ }^{13-16}$ The stochastic analysis was employed for statistical inference in a stochastic epidemic model for the Ebola virus in ${ }^{17}$. Furthermore, a discrete-time stochastic epidemic model with binomial distributions to study the transmission rate of the COVID-19 has been studied in Ref. ${ }^{18}$. The stochastic mathematical model for health care impact of the epidemic of novel coronavirus in India has been studied in ${ }^{19}$. Here, due to large uncertainly in the official data about the real cases numbers generated by the low number of tests made in countries as Brazil, the effect of randomness in the data is necessary and hence, the use of stochastic analysis 


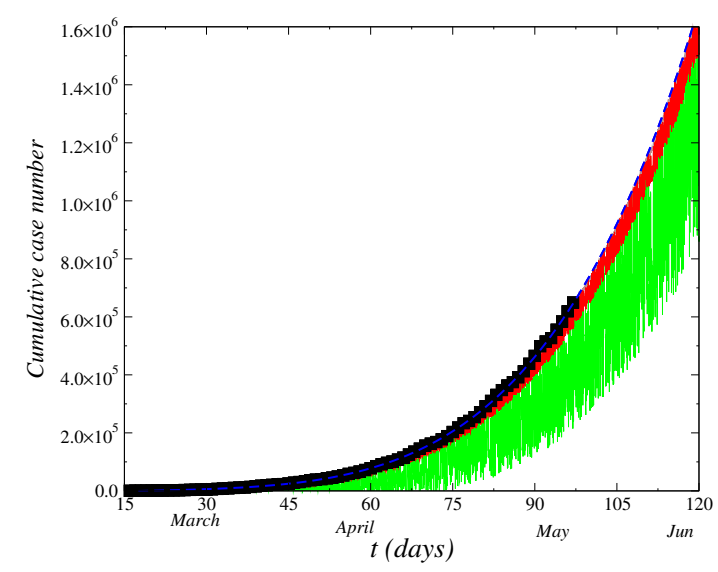

Figure 1. Effect of the stochastic term on cumulative total case number of the COVID-19 in Brazil. We perform the calculations for different values of $\beta_{0}$ in model Eq. (1) as $\beta_{0}=0.0$ (dashed blue-line), $\beta_{0}=3.0 \times 10^{-6}$ (red-line) and $\beta_{0}=5.0 \times 10^{-6}$ (green-line).

becomes largely adequate to treat the spreading of the disease. The plan of this paper is the following. In section 1, we describe the stochastic model. In section 2, we present the numerical results by stochastic differential equation. In section 3, we perform an analytical analysis, solving the correspondent Fokker-Planck equation. In section 4, we present our final remarks.

\section{A phenomenological Langevin equation for cumulative cases number dynamics}

The behavior of the cumulative total cases number $\mathscr{P}(t)$ of infected by coronavirus registered in the Brazil as function of time (days) from 15th March, 2020 is displayed in Fig. 1. The data were registered in the period. The non differential points are due to uncertainty in the official data and to population isolation conditions. Hence, for modeling of this behavior, we has added a randomly together with nonlinear terms in Eq. (1) with aim to simulate the effect of the uncertainty.

$$
d \mathscr{P}(t)=(f(t)+A(\mathscr{P}(t), t)) d t+B(\mathscr{P}(t), t) \circ d W
$$

where $f(t)$ is e polynomial of $n$ degree. Furthermore, we have $A(\mathscr{P}(t), t)$, given by $A(\mathscr{P}(t), t)=\alpha \mathscr{P}(t)(1-v \mathscr{P}(t))$ and $B(\mathscr{P}(t), t)=\beta_{0} t^{3}$ (multiplicative white noise) in Eq. (1) and the calculations were performed for different values of $\beta_{0}$ (constant). We obtain a strong oscillation of the curve with the increasing of $\beta_{0}$ indicating so, an trend of increase of growing of the uncertainly of the cumulative total case number. The data are considered from 15-March up to now.

\subsection{Phenomenological Model for novel cases}

The model of interest for spreading of novel cases of COVID-19 is given by the following stochastic model with a drift term in the form of a logistic model with a randomly term

$$
d N(t)=h+[g(t)-\alpha N(t)(1-v N(t))(1-\eta N(t))] d t+\beta(t) d \mathscr{W} .
$$

The deterministic part can adjust to the reported novel cases data in each day $t$ using a $n$ degree $(n \leq 6)$ polynomial. Furthermore, the assumption of a constant rate $h$ is reasonable when $N$ is large but becomes less so when $N$ is small. $\mathscr{W}(t)$ is the Wiener process or Brownian motion. We choice a multiplicative noise of the form $\beta_{0} t^{n}$, where $\beta_{0}$ is an arbitrary constant.The equation above describes a particle in Brownian motion under action of a nonlinear potential. ${ }^{20}$ Furthermore, one has a dissipating force given by $-\gamma \dot{x}$ represented by the drift term in the Langevin's equation and a white noise term $\xi(t)$, that relates to the Wiener process as $\mathscr{W}(t)$ by $\mathscr{W}(t)=\int_{t_{0}}^{t} \xi\left(t^{\prime}\right) d t^{\prime} ;\langle\xi(t)\rangle=0 ;\left\langle\xi(t) \xi\left(t^{\prime}\right)\right\rangle=\delta \Gamma\left(t-t^{\prime}\right)$. The dynamics of novel cases $N(t)$ of infected number by COVID-19 registered in the Brazil as function of time (days) registered in the period from 15th Mar, 2020 up to 12th Ago is displayed in Fig. 2. From fit of least minimums squares to the set of data supplied of the Brazilian agencies, we estimate the curve for the behavior of the novel cases given by a polynomial of $t$ (solid-line) in the Fig. 2 given by $g(t)=8191.2-620.92 t+12.63 t^{2}-0.05 t^{3}$. The data are up to Ago $12^{\text {th }}$. The zigzag behavior in the range of large $t$ values reflects in an increase of the uncertainty in the data and low-number of test performed. For modeling of this behavior, we have added a random noise term in the logistic model for growing of infectious disease as given by Eq. (2) with the aim to 


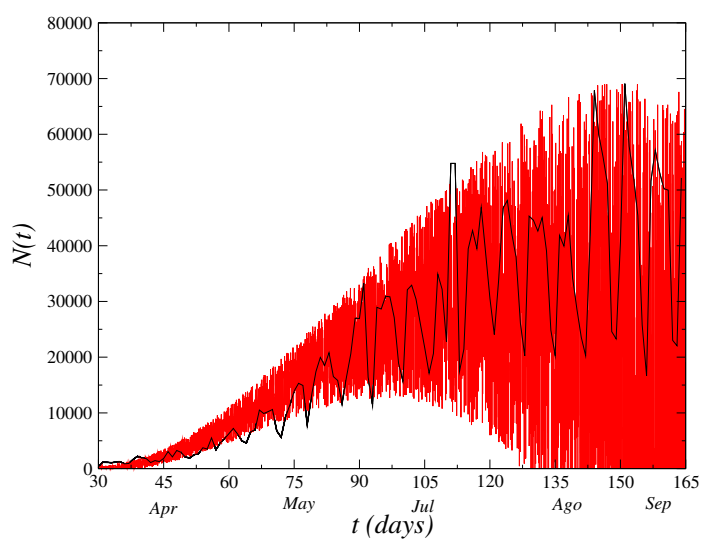

Figure 2. (Left) Dynamics of novel cases $N(t)$ in Brazil. The zigzag behavior in the results is reflected by the stochastic term in Eq. (2). We plot the time series of the model Eq. (2) for $\alpha=v=0.125, \eta=0.0625$ and noise amplitude $\Gamma=1$ as well as $\beta(t)=3.0 \times 10^{-6} t^{3}$.

simulate the effect of this uncertainty. The $\alpha, v$ and $\eta$ parameters comes from the logistic model with threshold for spreading of the smallpox with the value $\alpha=v=0.125$. Thus the range of $\eta$ employed is into $0<\eta v$. The polynomial $g(t)$ comes from the adjustment to the data within the range considered. As, both COVID-19 and smallpox, once contracted confers to the individuals a lifetime immunity, considering a group of individuals in a given time $t$ where one has a number of these individuals surviving a time $t$ later. Although the model was based on Brazilian data, it can apply to other countries as well, since $g(t)$ is a function that adjustments to the data set of COVID-19 of each country with a smaller uncertainty and that after a time $t$, there is a larger distance from the real data. In Fig. 3, we show the time evolution of novel cases $N(t)$ for some countries as United States, India and all world. The range of data considered is up to Ago 12th. The India data present a stronger oscillation into the range displayed in the figure, where in the beginning of the pandemic the oscillation is small (do not displayed in the figure). The effect of the stochastic term is to span the fluctuations of the data (as displayed in figs. 2 and 3) that in fact are not completely stochastic but follow a weekly cycle. These daily fluctuations with a weekly cycle in COVID reported cases have been observed in many countries and are mainly due to diagnostic and data reporting practices ${ }^{21}$ We find the time series of the model Eq. (2) in each case for the values $\alpha=v=0.125, \eta=0.0625$ and noise amplitude $\Gamma=1$. Furthermore, we use the additive white noise $\beta$ constant as: $\beta=2.0 \times 10^{6}$.

\subsection{Numerical Results}

We perform the simulation of the model Eq. (2) with the term $\beta \mathscr{W}(t)$, Wiener increment and multiplicative noise, whose standard deviation is given by $\sigma_{\mathscr{W}}=\sqrt{\Delta t}$. We write the Wiener increment as $\beta d \mathscr{W}(t) \sim \sqrt{d t} \beta R_{G}$, where $R_{G}$ is an aleatory generator number with Gaussian distribution of mean zero and variance $\sigma_{\mathscr{W}}^{2}=1$. In Fig. 2, we plot the time series of the model Eq. (2) for $\beta(t)=3.0 \times 10^{-6} t^{3}$. For all case analyzed, one has the time series of the variation of novel cases oscillating quickly as displayed in the figure.

In Fig. 4, we plot the half-width of probability density $P(N, t)$ as function of time $t, \sigma(t)$. We calculate the variance of the distribution where the standard deviation gives an estimating of novel cases number in each day. The results adjust to the official data of ministry of healthy within range considered. The difference to the real data may be due to approach used. A treatment considering a nonwhite noise approach $\zeta(t)$ (which approaches a delta-correlated noise) may give a better adjusts to the real data. Anyway, it seems to exists a large probability of increase in the novel cases number within the next weeks up to a plateaus. In Fig. 5 we plot the mean-square deviation root $\lambda_{N}=\sqrt{\left\langle N^{2}\right\rangle}=\sqrt{2 \beta(t)^{2} t}$ as function of $t$, with aim to calculate the analogous to the displacement $\lambda_{N}$ that a particle experiences on the average or, the square root of the arithmetic mean of the square of the displacement. This reflects the behavior that a particle in Brownian motion in the fluid where Einstein and Smoluchowski (on independent way) have derived a law (Einstein-Smoluchowski law) which is a manifestation of the fluctuation-dissipation theorem. As this law gives the mean-square deviation of the motion of the particle in suspension in the fluid $\left\langle x^{2}\right\rangle \propto T$ where $T$ is the temperature of the fluid, we must have an analogous relation for the mean-square deviation root of novel cases $\left\langle N^{2}\right\rangle$.

We introduce the $n$th order moments $\mu_{n}=\left\langle\left(x-m_{1}\right)^{n}\right\rangle$ about the mean or central moments, where we have the following relations: $c_{1}=\mu_{1}, c_{2}=\mu_{2}, c_{3}=\mu_{3}, c_{4}=\mu_{4}-3 \mu_{2}^{2}$. Normalized measures often used, indicating a deviation from a Gaussian, are the kurtosis $\lambda_{4}$, defined as $\lambda_{4}=\mu_{4} / \sigma^{4}-3$ and the skewness $\lambda_{3}$. In Fig. 6, we display the behavior of the kurtosis (excess), $\lambda_{4}(t) . \lambda_{4}(t)$ is numerically calculated (solving Eq. (30)). Furthermore, the kurtosis relates to the deviation of the tail of the distribution, as compared to Gaussian $P(N, t)=(1 / \sqrt{4 \pi \beta t}) e^{-N^{2} / 4 \beta t}$, whose solution would correspond to the Eq. (2) and 

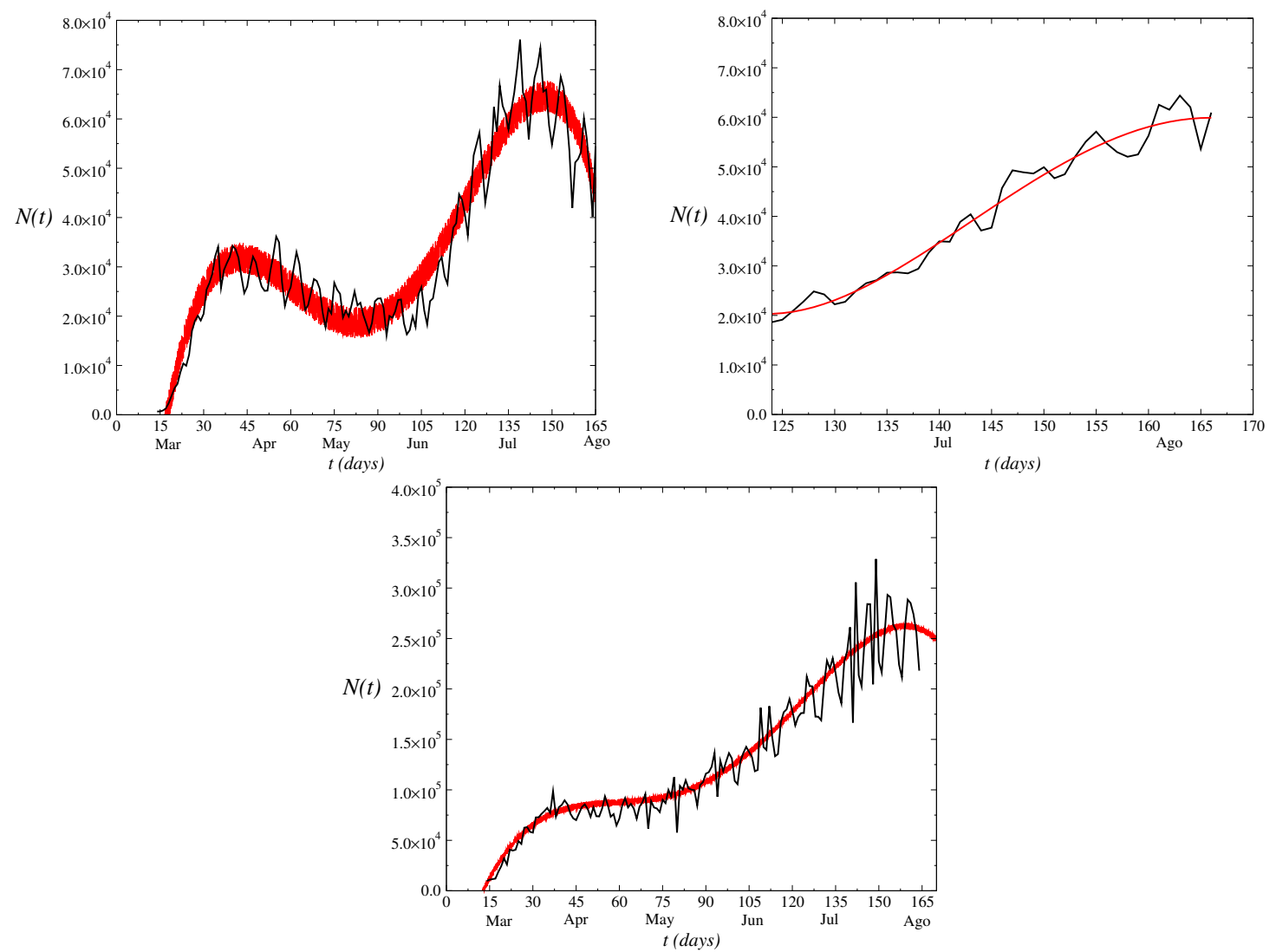

Figure 3. Dynamics of new cases $N(t)$ in United Sates (left), India (Center) and all world (right) and the correspondent time series obtained for each model Eq. (2). The data were obtained for values $\alpha=v=0.125, \eta=0.0625$ and noise amplitude $\Gamma=1$ and additive white noise: $\beta=2.0 \times 10^{6}$.

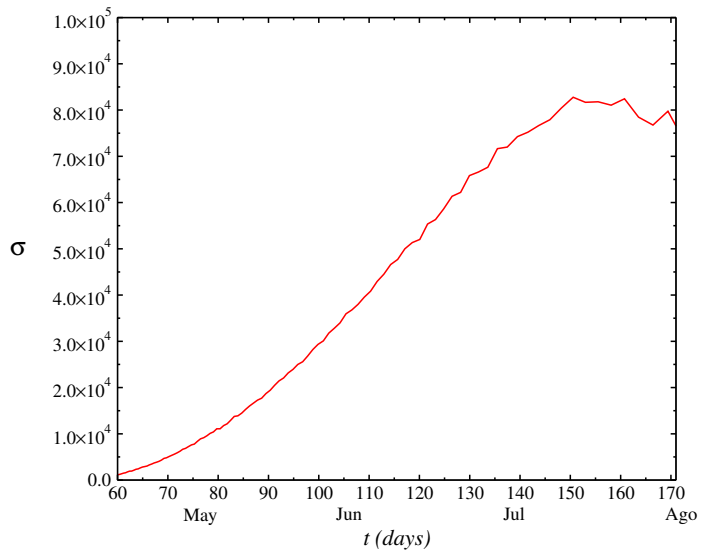

Figure 4. Behavior of the half-width of the distribution as a function of $t, \sigma(t)$. The half-width gives an expectation of new cases in each day $t$. 


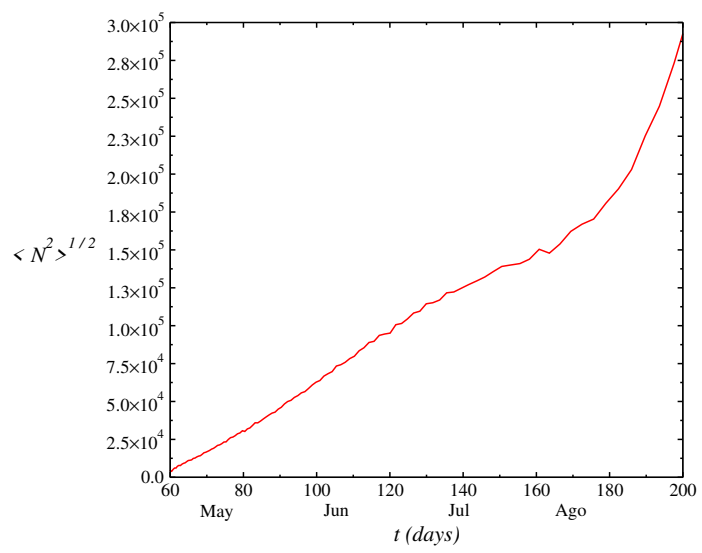

Figure 5. Mean-square root deviation $\lambda_{N}=\sqrt{\left\langle N^{2}\right\rangle}$ analogous to the mean-square root deviation of a particle in Brownian motion.

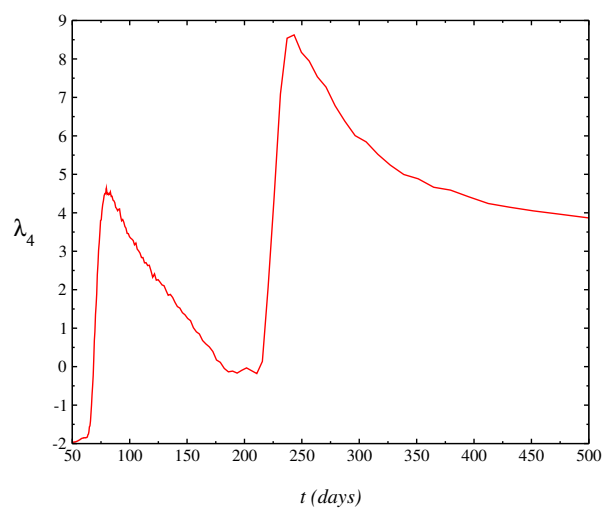

Figure 6. Behavior of the kurtosis as a function of $t \lambda_{4}(t)$. The range of negative values give an estimating of the shape of distribution which becomes nearest of a Gaussian for $\lambda_{4}=0$ at range of large $t$ values since the firsts cases reported in the Brazil on $15^{\text {th }}$ March 2020.

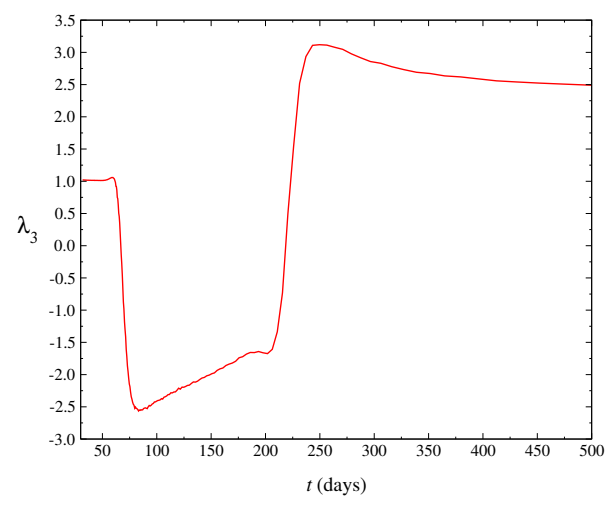

Figure 7. Behavior of the skewness as a function of $t \lambda_{3}(t)$. The negative (positive) values obtained give an estimating of the shape of distribution that becomes nearest of a Gaussian when $\lambda_{3} \rightarrow 0$ at range of large $t$ values since the firsts cases registered in Brazil on 15 th March 2020. 
(30)) for the $v=\eta=0$ and $g(t) \equiv 0$ cases. The range of negative values obtained for the kurtosis indicates that the shape of the distribution is next to the Wigner semicircle ${ }^{11}$. Furthermore, at range of small $t$ where the kurtosis is nearest to zero we have that the distribution is nearest of a Gaussian $\left(\lambda_{4}=0\right)$.In Fig. 7, we display the behavior of another statistical test: the skewness, $\lambda_{3}(t)=c_{3} / c_{2}^{3 / 2} . \lambda_{3}(t)$ gives a measure of the degree of asymmetry of the distribution. When skewness is zero, we have a perfect Gaussian. From result of the Figure, we find a large asymmetry in the distribution which must not obey to the Gaussian distribution.

\section{Nonlinear Fokker-Planck equation}

\subsection{Nonlinear Fokker-Planck equation within a Itô prescription}

The nonlinear Fokker-Planck equation is given in the non-additive statistical mechanics by

$$
\frac{\partial P(x, t)}{\partial t}=D \frac{\partial^{2}\left\{[P(x, t)]^{2-q}\right\}}{\partial x^{2}}-\frac{\partial[P(x, t) K(x, t)]}{\partial x} .
$$

We obtain that Eq. (3) is equivalent to following Itô stochastic differential equation

$$
d X=A(X(t), t) d t+\phi(X(t), t) \circ d W
$$

where $A(X(t), t)=K(X(t), t)$ and $\phi(X(t), t)=[P(X(t), t)]^{\frac{1-q}{2}} . X(t)$ is a stochastic process defined on probability space $\Omega$, being the triple $(\Omega, \mathscr{F}, P)$ a probability space, where $\mathscr{F}$ is a $\sigma$-algebra and $P$ a probability measure that is, a function that to every set $A \in \mathscr{F}$ assigns a number in range $[0,1]$, where $P(\Omega)=1, P(\emptyset)=0$ and

$$
P\left(\bigcup_{n=1}^{\infty} A_{n}\right)=\sum_{n=1}^{\infty} P\left(A_{n}\right)
$$

The random variable $X$, defined on $\Omega$ with the property that for every Borel subset $B$ of $\mathbb{R}$, the subset of $\Omega$ given by $\{X \in B\}=\{\omega \in \Omega ; X(\omega) \in B\}$ is in the $\sigma$-algebra $\mathscr{F}$. Moreover, $P(X(t), t)$, given $X$ a random variable on a probability space $(\Omega, \mathscr{F}, P)$ is the probability measure of $X . \mu_{X}$ assigns to each Borel subset $B$ of $\mathbb{R}$ the mass $\mu_{X}(B)=P\{X \in B\}^{9}$.

The Itô integral for $\phi$ is given by

$$
\int \phi(X(t), t) d W(t)=\mathrm{ms}-\lim _{n \rightarrow \infty}\left\{\sum_{j=1}^{n} \phi\left(t_{i-1}\right)\left[W\left(t_{i}\right)-W\left(t_{i-1}\right)\right]\right\},
$$

where ms- lim means square limit. $W(t)$ is a Markovian process, presenting normal distribution, satisfying the conditions $\langle\xi(t)\rangle=0$ and $\left\langle\xi(t) \xi\left(t^{\prime}\right)\right\rangle=\delta\left(t-t^{\prime}\right)$.

We can write the Eq. (3) as

$$
\frac{\partial P(x, t)}{\partial t}=D \frac{\partial^{2}\left\{[P(x, t) P(x, t)]^{1-q}\right\}}{\partial x^{2}}-\frac{\partial[P(x, t) K(x, t)]}{\partial x},
$$

where we define $A(x, t)=K(x, t)$ and $\phi(X(t), t)=[P(X(t), t)]^{\frac{1-q}{2}}$ to obtain the correspondent Itô stochastic differential equation

$$
d X=K(X(t), t) d t+[P(X(t), t)]^{\frac{1-q}{2}} \circ d W
$$

which is the Eq. (4). The solution for $X(t)$ using the Stratonovich integral is given as

$$
X(t)=X(0)+\int_{0}^{t} K\left(s, X_{s}\right) d s+\int_{0}^{t} \phi\left(s, X_{s}\right) d W_{s}
$$

This implies that $X(t)$ is the solution of the following modified Itô equation

$$
X(t)=X(0)+\int_{0}^{t} K\left(s, X_{s}\right) d s
$$

where $\phi^{\prime}$ denotes the derivative of $\phi(x, t)$ with respect to $x$. Therefore, the Eq. (3) in Itô calculus is different of the Stratonovich interpretation. 
From the Feynman-Kac theorem, let $h(x)$ be a Borel-measurable function. Fix $T>0$, and let $t \in[0, T]$ be given, we define the function

$$
g(x, t)=E^{t, x} h(X(T)),
$$

where

$$
E|g(X)|=\int_{-\infty}^{\infty}|g(x)| P(x) d x
$$

Furthermore, we assume $E^{t, x}|h(X(T))|<\infty$ for all $t$ and $x$, then $g(x, t)$ satisfies the partial differential equation

$$
\frac{\partial g(x, t)}{\partial t}+K(x, t) \frac{\partial g(x, t)}{\partial x}+\frac{1}{2} \phi^{2}(x, t) \frac{\partial^{2} g(x, t)}{\partial x^{2}}=0
$$

with the terminal condition $g(x, t)=h(x)$ for all $x$, where we assume that the stochastic process $g(X(t), t), 0 \leq t \leq T$ is a martingale. ${ }^{9}$.

From Eq. (4), we obtain the time development of an arbitrary $f(X(t))$ using the Itô formula ${ }^{8}$

$$
f[X(t)+d X(t)]-f[X(t)]=\frac{\partial f}{\partial x}\left[K(X(t), t) d t+[P(X(t), t)]^{\frac{1-q}{2}} \circ d W\right]+\frac{1}{2} \frac{\partial^{2} f}{\partial x^{2}}\left\{[P(X(t), t)]^{\frac{1-q}{2}}\right\}+\mathscr{O}(\cdots) .
$$

Taking the average of both sides in the equation above, we obtain

$$
\left\langle\frac{d f}{d t}\right\rangle=\left\langle\left[\frac{d f}{d x} K(X(t), t)+\frac{1}{2} \frac{\partial^{2} f}{\partial x^{2}}\left\{[P(X(t), t)]^{\frac{1-q}{2}}\right\}\right]\right\rangle+\frac{d}{d t}\left\langle\left\{[P(X(t), t)]^{\frac{1-q}{2}}\right\}\right\rangle
$$

and using

$$
\begin{array}{r}
\frac{\langle f(X(t)\rangle}{d t}=\frac{d}{d t} \int_{-\infty}^{\infty} d x f(x) P(x, t) \\
=\int_{-\infty}^{\infty} d x f(x) \frac{\partial}{\partial t}[P(x, t)]=\int_{-\infty}^{\infty} \frac{\partial f}{\partial x} K(x, t) P(x, t) d x+\frac{1}{2} \int_{-\infty}^{\infty} \frac{\partial^{2} f}{\partial x^{2}}\left\{[P(x, t)]^{\frac{1-q}{2}}\right\} P(x, t) d x+\int_{-\infty}^{\infty} d x f(x) \frac{\partial P}{\partial t} .
\end{array}
$$

We integrate by parts and discard surface terms to obtain

$$
\int_{-\infty}^{\infty} d x f(x) \frac{\partial}{\partial t}[P(x, t)]=\int_{-\infty}^{\infty} f(x) \frac{\partial}{\partial x}[K(x, t) P(x, t)] d x+\frac{1}{2} \int_{-\infty}^{\infty} f(x) \frac{\partial^{2}}{\partial x^{2}}\left[\left\{[P(x, t)]^{\frac{1-q}{2}}\right\} P(x, t)\right] d x
$$

and therefore

$$
\frac{\partial}{\partial t} P(x, t)=-\frac{\partial}{\partial x}[K(x, t) P(x, t)]+\frac{1}{2} \frac{\partial^{2}}{\partial x^{2}}\left\{\left\{[P(x, t)]^{\frac{1-q}{2}}\right\}^{2} P(x, t)\right\} .
$$

Thus, we have a complete equivalence between the diffusion process defined by a drift coefficient $K(x, t)$ and a diffusion coefficient given as $\phi(x, t)=[P(x, t)]^{1-q}$, in which the diffusion process can be locally approximated by an Itô stochastic differential equation.

Correspondence to Stratonovich stochastic differential equation

$$
(S) d X=K^{S}(X(t), t) d t+[P(X(t), t)]^{\frac{1-q}{2}} d W,
$$

with $K^{s}=K-\frac{1}{2} \phi \partial_{x} \phi$ and using the correspondence between the Itô stochastic differential equation and the Fokker-Planck equation, we have a equivalent Fokker-Planck equation

$$
\partial_{t} P=-\partial_{x}\left\{K^{S} P\right\}+\frac{1}{2} \partial_{x}\left\{\phi \partial_{x}[\phi P]\right\}
$$


which is known as Stratonovich form of the Fokker-Planck equation ${ }^{8}$. However, it is different from Eq. (3). Therefore, we have that the corresponding nonlinear Fokker-Planck equation in the Stratonovich prescription is different from nonlinear equation obtained in the Itô prescription, being the Itô stochastic differential equation more usually employed to make the connection with the Fokker-Planck equation. In spite of both definitions can be related by the choosing of $i$ by $\tau_{i}=\alpha t_{i}+(1-\alpha) t_{i-1}$, $\alpha \in(0,1)$ they generate different definitions of stochastic integral (Itô integral and Stratonovich )integral) and consequently to different stochastic differential equations, even though the Itô stochastic differential equation is equivalent to an another Stratonovich equation however, with an additional term ${ }^{8}$.

\subsection{Existence and Uniqueness}

We can investigate the existence and uniqueness of solutions of nonlinear differential equations utilizing the well-known existence and uniqueness theorem for stochastic differential equations ${ }^{9}$. Let $T>0$ and $K(x, t):[0, T] \times \mathbb{R}^{n} \rightarrow \mathbb{R}^{n}, \phi(x, t)$ : $[0, T] \times \mathbb{R}^{n} \rightarrow \mathbb{R}^{n \times m}$ be measurable functions satisfying

$$
|K(x, t)|+|\phi(x, t)| \leq C(1+|x|) ; x \in R^{n}, \quad t \in[0, T]
$$

for some constant $C$ and such that

$$
|b(x, t)-b(y, t)|+\mid\left(\phi(x, t)-\phi(y, t)|\leq D| x-y \mid ; \quad x, y \in \mathbb{R}^{n}, \quad t \in[0, T]\right.
$$

for some constant $D$. Let $Z$ be a random variable which is independent on the $\sigma$-algebra $\mathscr{F}_{\infty}^{m}$ generated by $W_{s}(\cdot), s \leq 0$ and such that the expectation $E\left[|Z|^{2}\right]<\infty$. Then the stochastic differential equation

$$
d X=A(X(t), t) d t+\phi(X(t), t) d W, \quad 0 \leq t \leq T, X_{0}=Z
$$

has a unique $t$-continuous solution $X_{t}(\omega)$ with the property that $X_{t}(\omega)$ is adapted to filtration $\mathscr{F}_{t}^{Z}$ generated by $Z$ and $W_{s}(\cdot)$; $s \leq t$

$$
E\left[\int_{0}^{T}\left|X_{t}\right|^{2} d t\right]<\infty
$$

\section{Analytical Results by Fokker-Planck equation}

We can perform an analytical analysis solving the correspondent Fokker-Planck equation to the stochastic differential equation Eq. (2). We start from the time development of an arbitrary function of the stochastic process $N(t), f(N(t))$, using the Itô formula

$$
f[N(t)+d N(t)]-f[N(t)]=\partial_{N} f[N(t)]\left\{\left[g\left(t^{\prime}\right)-\alpha N\left(t^{\prime}\right)\left(1-v N\left(t^{\prime}\right)\right)\left(1-\eta N\left(t^{\prime}\right)\right)\right] d t+\beta d \mathscr{W}\right\}+\frac{\beta^{2}}{2} \partial_{N}^{2} f[N(t)](d \mathscr{W})^{2}
$$

where the higher order terms have been discarded, and $(d \mathscr{W}(t))^{2}=d t$. Taking the average of both sides in the equation above, we find

$$
\left\langle\frac{\partial f}{\partial t}\right\rangle=\left\langle\left[\frac{\partial f}{\partial x}\{[g(t)-\alpha x(1-v x)(1-\eta x)] d t+\beta d \mathscr{W}\}+\frac{\beta^{2}}{2} \frac{\partial^{2} f}{\partial x^{2}}\right]\right\rangle .
$$

In following, using

$$
\begin{array}{r}
\frac{d}{d t}\langle f(N(t))\rangle=\frac{d}{d t} \int_{-\infty}^{\infty} d x f(x) P(x, t)=\int_{-\infty}^{\infty} d x f(x) \frac{\partial}{\partial t}[P(x, t)]=\int_{-\infty}^{\infty} \frac{\partial f}{\partial x}\{[g(t)-\alpha x(1-v x)(1-\eta x)]\} P(x, t) d x \\
+\frac{\beta^{2}}{2} \int_{-\infty}^{\infty} \frac{\partial^{2} f}{\partial x^{2}} P(x, t) d x
\end{array}
$$

we integrate by parts and discard surface terms to find

$$
\int_{-\infty}^{\infty} d x f(x) \frac{\partial}{\partial t}[P(x, t)]=\int_{-\infty}^{\infty} f(x) \frac{\partial}{\partial x}\{[g(t)-\alpha x(1-v x)(1-\eta x)] P(x, t)\} d x+\frac{\beta^{2}}{2} \int_{-\infty}^{\infty} f(x) \frac{\partial^{2}}{\partial x^{2}}[P(x, t)] d x .
$$

and hence

$$
\frac{\partial}{\partial t} P(x, t)=-\frac{\partial}{\partial x}\{[g(t)-\alpha x(1-v x)(1-\eta x)] P(x, t)\}+\frac{\beta^{2}}{2} \frac{\partial^{2}}{\partial x^{2}} P(x, t) .
$$


Therefore, the associated Fokker-Planck equation correspondent to the stochastic model Eq. (30) is given by

$\frac{\partial P(x, t)}{\partial t}=\frac{\partial}{\partial x}\{[g(t)-\alpha x(1-v x)(1-\eta x)] P(x, t)\}+\frac{\beta^{2}}{2} \frac{\partial^{2}[P(x, t)]}{\partial x^{2}}$.

taking the Fourier transform of the above equation, we can guarantee the normalization of the probability density where $P(x)$ is well behaved. We take the boundaries at infinity as $\lim _{x \rightarrow \infty} P(x, t)=0$ and $\partial_{x} P(x)$ being reasonably well behaved. As $\lim _{x \rightarrow \infty} \partial_{x} P(x, t)=0$ so, a nonzero current of probability at infinity will usually require that the terms in the equation above will become infinite there ${ }^{8}$. We use the initial condition $P\left(x_{0}, 0\right)=P_{0}$.

For solving the Fokker-Plank equation independent on time, we make a power series expansion $P(x, t)=\sum_{n=0}^{\infty} a_{n}(t) x^{n}$ to find

$\frac{\partial P}{\partial t}=\alpha(1-2 v x)(1-\eta x) P-[g(t)-\alpha x(1-v x)(1-\eta x)] \frac{\partial P}{\partial x}+\frac{\beta^{2}}{2} \frac{\partial^{2} P}{\partial x^{2}}$
$\sum_{n=0}^{\infty}\left(\frac{d a_{n}}{d t}+g(t)(n+1) a_{n+1}\right) x^{n}=[\alpha(1-2 v x)(1-\eta x)] \sum_{n=0}^{\infty} a_{n} x^{n}+\alpha x(1-v x)(1-\eta x) \sum_{n=0}^{\infty} n a_{n} x^{n-1}+\frac{\beta^{2}}{2} \sum_{n=0}^{\infty} n(n-1) a_{n} x^{n-2}$.

where we obtain the following recurrence relations

$$
\alpha(1-2 v x)(1-\eta x) \sum_{n=0}^{\infty} a_{n} x^{n}+\alpha x(1-v x)(1-\eta x) \sum_{n=1}^{\infty}(n+1) a_{n+1} x^{n}+\frac{\beta^{2}}{2} \sum_{n=0}^{\infty}(n+1)(n+2) a_{n+2} x^{n}=k,
$$

where $k$ is a separation constant. For $k=0$, one obtains the following other recurrence relations

$$
a_{2}=-\frac{\alpha}{\beta^{2}} a_{0}, \quad a_{3}=-\frac{\alpha}{3 \beta^{2}} a_{1}, \quad a_{4}=\frac{\alpha^{2}}{6 \beta^{4}} a_{0}, \quad a_{5}=\frac{\alpha^{2}}{30 \beta^{4}} a_{1}
$$

Additionally, we have

$$
\frac{d a_{n}(t)}{d t}+g(t)(n+1) a_{n+1}(t)=k
$$

Therefore, we obtain $P(x)$ in the form

$P(x)=a_{0}\left(1-\frac{\alpha}{\beta^{2}} x^{2}+\frac{\alpha^{2}}{\beta^{4}} x^{4}+\cdots\right)+a_{1}\left(x-\frac{\alpha}{3 \beta^{2}} x^{3}+\frac{\alpha^{2}}{30 \beta^{4}} x^{5} \cdots\right)$

where the constants $a_{0}$ and $a_{1}$ are determined by the initial conditions $P(0,0)=P_{0}$ and $\partial_{x} P(x, 0)=0 \mathrm{em} x=0$. We find $a_{0}=P_{0}$ and $a_{1}=0$. From the normalization condition, the second term in the density probability above must be zero and hence, all coefficients $a_{1}$ must cancel. Therefore, we have

$$
P(x, t)=P_{0}\left(1-\frac{\alpha}{\beta^{2}} x^{2}+\frac{\alpha^{2}}{\beta^{4}} x^{4}+\cdots\right)
$$

To ensure the normalization of the probability density, $P_{0}$ must be non zero only within the interval $-\varepsilon \leq x \leq \varepsilon$ and zero out it.

For $k \neq 0$, we have from Eq. (32) that $n=0$ and $a_{2}+\alpha a_{0} / \beta^{2}=k$ and all $a_{n}$ higher are zero. Hence, we find from integration of the Eq. (34)

$$
P(t)=\frac{\beta^{2}}{\alpha} P_{0} t\left[1-a_{2}(t)\right]+a_{0}(0)-\int_{0}^{t} g\left(t^{\prime}\right) a_{1}\left(t^{\prime}\right) d t^{\prime} .
$$

We find the $n$th moments $m_{n}=\left\langle N^{n}\right\rangle=\int_{-\infty}^{\infty} N^{n} P(N, t) d N$, where the mean half-width of the probability distribution $\sigma=\sqrt{\left\langle N^{2}\right\rangle-\langle N\rangle^{2}}$ gives an estimating of the novel cases in the day $t$. From the solution of the Fokker-Planck equation we find the analytical expression of the mean half-width of the distribution as function of time given by

$$
\sigma(t)=\left[\frac{\gamma}{\alpha} P_{0} t\left(1-a_{2}(t)\right)+a_{0}(0)-\int_{0}^{t} g\left(t^{\prime}\right) a_{1}\left(t^{\prime}\right) d t^{\prime}\right] \sqrt{\frac{[N(t)]^{3}}{3}-\frac{[N(t)]^{4}}{4}}
$$


Furthermore, we have $(n=0) d a_{0}(t) / d t=0$ and so $a_{0}(t)=c, a_{1}=k f(t) / 2$ and $P(t)=P_{0} t+c-k / 2$, where we define $p_{0}=c-k / 2$. Therefore, we have

$$
\sigma(t)=\left(P_{0} t+p_{0}\right) \sqrt{\frac{[N(t)]^{3}}{3}-\frac{[N(t)]^{4}}{4}} .
$$

How the first cases are registered on March 15th $(t=15)$, we obtain $p_{0}=-1.5$ an $P_{0}=0.1$ for the official results of the novel cases numbers to obtain a concordance with the numerical results of the stochastic analysis.

\section{Conclusions}

In Brief, we propose a stochastic model for the time evolution of the SARS-CoV-2 (COVID-19) in Brazil based in the nonlinear Itô's diffusion. Our results are compared with official data supplied by the Brazilian healthy agencies where due to large uncertain in the results generated principally by the low number of tests made in the population and hence, to under reporting, generates a large uncertainly in the official results and consequently, the stochastic differential equation analysis becomes the more realistic model for growing of the total number of infected $\mathscr{P}$ and novel cases number $N(t)$. We can use an approach beyond white noise limit to try to better describe the expansion dynamics what can be done in a future work. The model reported here is based on Brazilian data from March 15 which shows an upward trend in the coming weeks.

\section{References}

1. Giordano, G. et al. Modelling the covid-19 epidemic and implementation of population-wide interventions in italy. Nat. Medicine 26, 855-860, DOI: doi.org/10.1038/s41591-020-0883-7 (2020).

2. Gattoa, M. et al. Modelling the covid-19 epidemic and implementation of population-wide interventions in italy. PNAS 117, 10484-10491, DOI: doi:10.1073/pnas.2004978117/-/DCSupplemental.y (2020).

3. Fanelli, D. \& Piazza, F. Analysis and forecast of covid-19 spreading in china, italy and france. Chaos Solitons Fractals 135, 109794, DOI: doi:10.1016/j.chaos.2020.109794 (2020).

4. Boccaletti, S., Ditto, W., Mindin, G. \& Antangana, A. Modeling and forecasting of epidemic spreading: The case of covid-19 and beyond. Chaos Solitons Fractals 134, 109761-109767, DOI: https://doi.org/10.1016/j.chaos.2020.109761 (2020).

5. Ndairou, F., Area, I., Nieto, J. J. \& Torres, D. F. M. Modeling and forecasting of epidemic spreading: The case of covid-19 and beyond. Chaos Solitons Fractals 135, 109846, DOI: https://doi.org/10.1016/j.chaos.2020.109846 (2020).

6. Reif, F. Fundamentals of statistical and thermal physics (165).

7. W. E Boyce, R. C. D. Elementary differential equations (2012).

8. Gardiner, C. Stochastic methods, a handbook for the natural and social sciences (2009).

9. Øksendal, B. Stochastic differential equations an introduction with applications (2013).

10. Strogatz, S. H. Nonlinear dynamics and chaos (1994).

11. P. Richmond, S. H., J. Mimkes. Econophysics and physical economics (2013).

12. Jacobs, K. Stochastic processes for physicists (2013).

13. Lima, L. S. \& Miranda, L. L. B. Price dynamics of the financial markets using the stochastic differential equation for a potential double well. Phys. A 490, 828-833, DOI: https://doi.org/10.1016/j.physa.2017.08.106 (2018).

14. Lima, L. S., Oliveira, S. C., F., A. A. \& Melgaço, J. H. C. Breaks down of the modeling of the financial market with addition of non-linear terms in the ito stochastic process. Phys. A 526, 120932, DOI: https://doi.org/10.1016/j.physa.2019.04.168 (2019).

15. Lima, L. S. \& Santos, G. K. C. Stochastic process with multiplicative structure for the dynamic behavior of the financial market. Phys. A 512, 222-229, DOI: https://doi.org/10.1016/j.physa.2018.08.049 (2018).

16. Lima, L. S., Oliveira, S. C., Abeilice, A. F. \& Melgaço, J. H. C. Breaks down of the modeling of the financial market with addition of non-linear terms in the itô stochastic process. Phys. A 526, 120932, DOI: https://doi.org/10.1016/j.physa.2019. 04.168 (2019).

17. Lekone, P. \& Finkensta, B. Statistical inference in a stochastic epidemic seir model with control intervention: Ebola as a case study. Biometrics 62, 1170, DOI: https://doi.org/10.1111/j.1541-0420.2006.00609.x (2006). 
18. Lekone, P. \& Finkensta, B. A discrete stochastic model of the covid-19 outbreak: Forecast and control. Math. Biosci. Eng. 17, 2792-2804, DOI: doi:10.3934/mbe.2020153 (2020).

19. He, S., Tang, S. \& Rong, L. Healthcare impact of covid-19 epidemic in india: A stochastic mathematical model. Med. J. Armed Forces India 76, 147-155, DOI: https://doi.org/10.1007/s100510050582 (2020).

20. Bouchaud, J.-P. \& Cont, R. A langevin approach to stock market fluctuations and crashes. Eur. J. Phys. B 6, 543-550, DOI: https://doi.org/10.1007/s100510050582 (1998).

21. Bergman, A., Sella, Y. \& P. Agre, A. C. Oscillations in u.s. covid-19 incidence and mortality data reflect diagnostic and reporting factors. Clin. Sci. Epidemiol. 5, e00544-20, DOI: 10.1128/mSystems.00544-20 (2020).

\section{Acknowledgements}

This work was partially supported by the Brazilian agency CNPq.

\section{Author contributions statement}

Leonardo S. Lima is the sole author of this manuscript. He has conducted the experiment, analysed the results and written the paper. 